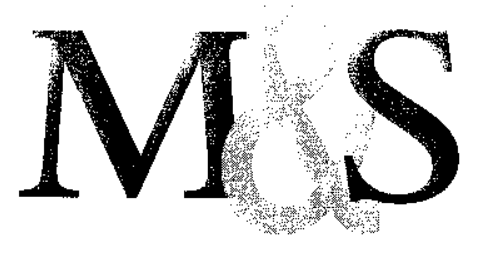

\title{
Materials and Structures
}

\section{Matériaux \\ et Constructions}

\author{
Surendra P. Shah \\ Editor-In-Chief \\ J. Marchand, S. Mindess, H. W. Reinhardt, A. Skarendahl \\ Associate Editors
}

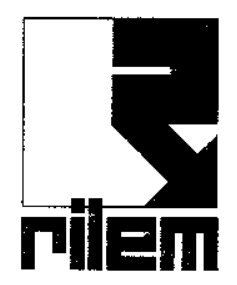




\title{
L'emploi de métakaolin dans la production de béton écologiquement efficace (The use of metakaolin to produce the eco-efficient concrete)
}

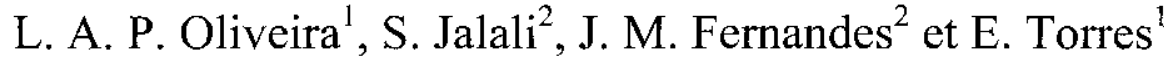 \\ (1) Département de Génie Civil, Université de Beira Interior, Covilhã, Portugal \\ (2) Département de Génic Civil, Université de Minho, Guimarães, Portugal
}

Received: 9 February 2004; accepted: 11 August 2004

\begin{abstract}
RÉSUME
Les matériaux d'addition font actuellement partie des développements les plus récents dans la production du béton, car l'utilisation des additions apporte une amélioration des propriétés mécaniques et de la durabilité du béton. D'autre part, leur utilisation a pour objectif de réduire la consommation de ciment, en contribuant de manière simple et économique à résoudre les problèmes liés à l'environnement. Cet article étudie les avantages et les limitations de la substitution partielle du ciment par le métakaolin. Les résultats obtenus démontrent que cette substitution apporte, pour le béton de même ordre de résistance à la compression, une amélioration considérable au niveau de la durabilité. Ce qui pennet de conclure que le métakaolin est un substitut efficace capable de réduire le contenu de ciment pour l'obtention de bétons équivalents.
\end{abstract}

1359-5997 (C) 2004 RILEM. All rights reserved.

\section{ABSTRACT}

One of the more recent developments in the production of concrete is the use of additions, resulting in a significant improvement to the mechanic and durability performances of concrete. Furthermore, to the extent that it leads to a reduction in cement consumption, the use of additions contributes to solving environmental problems in a simple and economic manner. This article presents a studv of the advantages and limitations of the partial replacement of cement by metakaolin in concrete. Its results demonstrate that such a substitution provides considerable improvements at the level of durability for concretes of the same level of compressive strength. This indicates that metakaolin is indeed an efficient substitute to cement, and its use results in the production of equivalent concretes.

\section{INTRODUCTION}

L'industrie de la construction est sans doute une des plus grandes consommatrices de ressources naturelles et est en même temps responsable de la production d'un grand volume de déchets. Au delà de cette constatation, un nouveau problème est apparu ces 30 dernières années : la dégradation précoce des structures en béton. Donc, la durabilité du béton et les questions liées à l'environnement sont les préoccupations fréquentes des études actuelles sur les bétons. Avec le développement des adjuvants superplastifiants et la disponibilité d'additions actives, l'emploi du béton de haute performance a fortement augmenté dans ces dernières années. L'un des matériaux les plus récents utilisés comme addition, remplaçant une partie du ciment dans les bétons, est le métakaolin. Son efficacité dans l'amélioration des propriétés du béton est prouvée par plusieurs études en laboratoire et par les résultats sur chantiers.

Les bétons contenant du métakaolin présentent des comportements similaires à ceux contenant de la fumée de silice, c'est-à-dire une amélioration de la résistance mécanique et une réduction significative de la perméabilité $[1,2]$. Cette addition entraîne aussi une réduction du retrait du béton $[3,4]$.

Certains auteurs [5-8] ont constaté qu'une addition de l'ordre de $15 \%$ de métakaolin confère une capacité supérieure de résistance en milieux agressifs, tels ceux des eaux de mer et des solutions acides.

L'utilisation d'additions actives dans les bétons comme substitut du ciment a des avantages divers dont les principaux se rapportent au fait que le ciment est le composant le plus coûteux du béton, que sa production exige une grande consommation d'énergie et que la production d'une tonne de ciment libère approximativement autant de dioxyde de carbone dans l'atmosphère [9].

Cet article présente une étude dont le but principal est de viabiliser l'utilisation de métakaolin produit à partir de kaolinites portugaises dans la production des bétons de haute performance considérés 'comme écologiquement efficaces. L'absence, au Portugal, de métakaolin fait que l'un des objectifs de ce travail fut la production et l'évaluation du métakaolin comme substitut partiel du ciment dans les bétons. Car il est connu qu'une réaction directe du métakaolin avec 


\begin{tabular}{|c|c|c|c|c|c|c|c|c|}
\hline \multicolumn{10}{|c|}{ Tableau 3 - Compositions et consistance des bétons } \\
\hline Matériaux & C300 & C30M10 & C330 & C33M10 & C33M15 & C33HRM10 & C33SF 10 & C400 \\
\hline Ciment $\left[\mathrm{kg} / \mathrm{m}^{3}\right]$ & 300 & 270 & 330 & 300 & 285 & 300 & 300 & 400 \\
\hline Rapport E/C $[\mathrm{kg} / \mathrm{kg}]$ & 0,35 & 0,35 & 0,45 & 0,45 & 0,45 & 0,45 & 0,45 & 0,4 \\
\hline Sable $\left[\mathrm{kg} / \mathrm{m}^{3}\right]$ & 1136 & 1136,3 & 1029,5 & 1029,5 & 1029,5 & 1029,5 & 1029,5 & 884,0 \\
\hline Pierre concassée $\left[\mathrm{kg} / \mathrm{m}^{3}\right]$ & 712,5 & 712,5 & 723,1 & 723,1 & 723,1 & 723,1 & 723,1 & 776,3 \\
\hline Métakaolin $\left[\mathrm{kg} / \mathrm{m}^{3}\right]$ & $\ldots$ & 30 & $\ldots$ & 30 & 45 & $\ldots$ & $\ldots$ & $\ldots$ \\
\hline SF $\left[\mathrm{kg} / \mathrm{m}^{3}\right]$ & $\ldots$ & $\ldots$ & $\ldots$ & $\ldots$ & $\ldots$ & $\ldots$ & 30 & $\ldots$ \\
\hline HRM $\left[\mathrm{kg} / \mathrm{m}^{3}\right]$ & $\ldots$ & $\ldots$ & $\ldots$ & $\ldots$ & $\ldots$ & 30 & $\ldots$ & $\ldots$ \\
\hline Rheobuild $[\%]$ & 2 & 2,5 & 1,5 & 2 & 2,5 & 1,75 & 1,75 & 1,5 \\
\hline Affaissement & 30 & 25 & 150 & 140 & 160 & 165 & 165 & 160 \\
\hline Cone d'Abrams $[\mathrm{mm}]$ & & & & & & & & $\ldots$ \\
\hline
\end{tabular}

mesurées. Le Tableau 3 montre les compositions des 8 mélanges de béton étudiés dans ce travail.

\section{PROTOCOLE D'ESSAIS}

Les déterminations de la résistance à la compression, de la résistivité électrique, de la diffusion de ions chlore et de l'absorption d'eau par capillarité ont été réalisées sur des échantillons de différents mélanges de béton à l'âge de 28 , 56 et 90 jours. Les valeurs présentées, pour tous les essais, sont les moyennes obtenues des résultats individuels de trois éprouvettes.

\subsection{Essais de résistance à la compression}

Les essais pour la détermination de la résistance à la compression ont été réalisés en conformité avec la norme LNEC E226 [14]. La vilesse d'application de la charge a été de $10 \mathrm{kN} / \mathrm{s}$ et les essais ont été réalisés sur des éprouvettes cubiques de $10 \mathrm{~cm}$ de côté.

\subsection{Essais de dịfusion des ions chlore}

La méthode "CTH Rapid Method», développée par Luping [15] a'été employée pour la détermination du coefficient de diffusion des ions chlore. Cette méthode consiste à soumettre une éprouvette cylindrique à une différence de potentiel pendant une période de temps préalablement établie. Une face de l'éprouvette a été mise en contact avec une solution cathode, contenue dans un compartiment amont, préparée avec $4 \mathrm{~g}$ de $\mathrm{NaOH}+100 \mathrm{~g}$ de $\mathrm{NaCl}$ par litre d'eau distillée et l'autre face en contact avec une solution anode, préparée avec $12 \mathrm{~g}$ de $\mathrm{NaOH}$ par litre d'eau, contenue dans un compartiment aval. Une fois le temps terminé, en fonction du courant initial, l'éprouvette est divisée axialement et la profondeur de pénétration est mesurée par un procédé colorimétrique, utilisant du nitrate d'argent. La différence de potentiel appliquée a été de $30 \pm 0,2 \mathrm{~V}$. La durée de l'essai est basée sur une proposition de Luping. Cette proposition, présentée au Tableau 4, a été revue et adaptée à ce travail.

\begin{tabular}{|c|c|}
\hline Tableau 4-Durée des essais CTH Rapid Method \\
\hline $\begin{array}{c}\text { Courant Initial } \\
\mathbf{I}_{0}[\mathbf{m A}]\end{array}$ & $\begin{array}{c}\text { Durée de l'essai } \\
\mathbf{t}_{\mathrm{cth}}[\text { heures] }\end{array}$ \\
\hline $\mathrm{I}_{0}<5$ & $\mathrm{t}_{\mathrm{cth}}=168$ \\
\hline $5 \leq \mathrm{I}_{0}<10$ & $\mathrm{t}_{\mathrm{chh}}=96$ \\
\hline $10 \leq \mathrm{I}_{0}<30$ & $\mathrm{t}_{\mathrm{cth}}=48$ \\
\hline $30 \leq \mathrm{I}_{0}<60$ & $\mathrm{t}_{\mathrm{chh}}=24$ \\
\hline $60 \leq \mathrm{I}_{0}<120$ & $\mathrm{t}_{\mathrm{cth}}=8$ \\
\hline $120 \leq \mathrm{I}_{0}<240$ & $\mathrm{t}_{\mathrm{cth}}=4$ \\
\hline $\mathrm{I}_{0} \geq 240$ & $\mathrm{t}_{\mathrm{cth}}=2$ \\
\hline
\end{tabular}

Le coefficient de diffusion est déterminé par l'expression suivante [15]:

$$
\begin{gathered}
D=\frac{R \cdot T \cdot L}{z \cdot F \cdot U} \cdot \frac{x_{d}-\alpha \cdot \sqrt{x_{d}}}{t} \\
\alpha=2 \cdot \sqrt{\frac{R \cdot T \cdot L}{z \cdot F \cdot U}} \cdot \operatorname{erf}^{-1} \cdot\left(1-\frac{2 \cdot c_{d}}{c_{0}}\right)
\end{gathered}
$$

Où:

D: coefficient de diffusion, $\mathrm{m}^{2} / \mathrm{s}$;

$z$ : valeur absolue de valence du ion, pour les ions de chlore $\mathrm{z}=1$;

$\mathrm{F}$ : paramètre de Faraday, $\mathrm{F}=9,648 \times 10^{4} \mathrm{~J} /(\mathrm{V} . \mathrm{mol})$;

$\mathrm{U}$ : valeur absolue de la différence de potentiel, $\mathrm{V}$;

$\mathrm{T}$ : température de la solution, $\mathrm{K}$;

$\mathrm{L}$ : épaisseur de l'éprouvette, $\mathrm{m}$;

$x_{d}$ : profondeur de pénétration, $\mathrm{m}$;

t: durée de l'essai, s;

$e r f^{-1}:$ l'inverse de la fonction d'erreur;

$c_{d}$ : concentration des ions chlore avec laquelle la couleur est modifiée, $c_{d} \approx 0,07 \mathrm{~N}$;

$c_{0}$ : concentration des ions chlore dans la cellule, $\mathrm{N}$.

\subsection{L'essai de résistivité électrique}

La résistivité électrique est déterminée par la lecture initiale du courant dans l'essai de diffusion des ions chlore. 


\begin{tabular}{|c|c|c|c|c|c|c|c|c|}
\hline \multicolumn{10}{|c|}{ Tableau 3-Compositions et consistance des bétons } \\
\hline Matériaux & C300 & C30M10 & C330 & C33M10 & C33M15 & C33HRM10 & C33SF10 & C400 \\
\hline Ciment $\left[\mathrm{kg} / \mathrm{m}^{3}\right]$ & 300 & 270 & 330 & 300 & 285 & 300 & 300 & 400 \\
\hline Rapport $\mathrm{E} / \mathrm{C}[\mathrm{kg} / \mathrm{kg}]$ & 0,35 & 0,35 & 0,45 & 0,45 & 0,45 & 0,45 & 0,45 & 0,4 \\
\hline Sable $\left[\mathrm{kg} / \mathrm{m}^{3}\right]$ & 1136 & 1136,3 & 1029,5 & 1029,5 & 1029,5 & 1029,5 & 1029,5 & 884,0 \\
\hline Pierre concassée $\left[\mathrm{kg} / \mathrm{m}^{3}\right]$ & 712,5 & 712,5 & 723,1 & 723,1 & 723,1 & 723,1 & 723,1 & 776,3 \\
\hline Métakaolin $\left[\mathrm{kg} / \mathrm{m}^{3}\right]$ & $\ldots$ & 30 & $\ldots$ & 30 & 45 & $\ldots$ & $\ldots$ & $\ldots$ \\
\hline SF $\left[\mathrm{kg} / \mathrm{m}^{3}\right]$ & $\ldots$ & $\ldots$ & $\ldots$ & $\ldots$ & $\ldots$ & $\ldots$ & 30 & $\ldots$ \\
\hline HRM $\left[\mathrm{kg} / \mathrm{m}^{3}\right]$ & $\ldots$ & $\ldots$ & $\ldots$ & $\ldots$ & $\ldots$ & 30 & $\ldots$ & $\ldots$ \\
\hline Rheobuild $[\%]$ & 2 & 2,5 & 1,5 & 2 & 2,5 & 1,75 & 1,75 & 1,5 \\
\hline $\begin{array}{c}\text { Affaissement } \\
\text { Cône d'Abrams [mm] }\end{array}$ & 30 & 25 & 150 & 140 & 160 & 165 & 165 & 160 \\
\hline
\end{tabular}

mesurées. Le Tableau 3 montre les compositions des 8 mélanges de béton étudiés dans ce travail.

\section{PROTOCOLE D'ESSAIS}

Les déterminations de la résistance à la compression, de la résistivité électrique, de la diffusion de ions chlore et de l'absorption d'eau par capillarité ont été réalisées sur des échantillons de différents mélanges de béton à l'âge de 28 , 56 et 90 jours. Les valeurs présentées, pour tous les essais, sont les moyennes obtenues des résultats individuels de trois éprouvettes.

\subsection{Essais de résistance à la compression}

Les essais pour la détermination de la résistance à la compression ont été réalisés en conformité avec la norme LNEC E226 [14]. La vitesse d'application de la charge a été de $10 \mathrm{kN} / \mathrm{s}$ et les essais ont été réalisés sur des éprouvettes cubiques de $10 \mathrm{~cm}$ de côté.

\subsection{Essais de diffusion des ions chlore}

La méthode "CTH Rapid Method», développée par Luping [15] a'été employée pour la détermination du coefficient de diffusion des ions chlore. Cette méthode consiste à soumettre une éprouvette cylindrique à une différence de potentiel pendant une période de temps préalablement établie. Une face de l'éprouvette a été mise en contact avec une solution cathode, contenue dans un compartiment amont, préparée avec $4 \mathrm{~g}$ de $\mathrm{NaOH}+100 \mathrm{~g}$ de $\mathrm{NaCl}$ par litre d'eau distillée et l'aúutre face en contact avec une solution anode, préparée avec $12 \mathrm{~g}$ de $\mathrm{NaOH}$ par litre d'eau, contenue dans un compartiment aval. Une fois le temps terminé, en fonction du courant initial, l'éprouvette est divisée axialement et la profondeur de pénétration est mesurée par un procédé colorimétrique, utilisant du nitrate d'argent. La différence de potentiel appliquée a été de $30 \pm 0,2 \mathrm{~V}$. La durée de l'essai est basée sur une proposition de Luping. Cette proposition, présentée au Tableau 4 , a été revue et adaptéc à ce travail.

\begin{tabular}{|c|c|}
\hline $\begin{array}{c}\text { Courant Initial } \\
\quad \mathbf{I}_{0}[\mathbf{m A}] \\
\end{array}$ & $\begin{array}{c}\text { Durée de l'essai } \\
t_{c t h} \text { [heures] }\end{array}$ \\
\hline$I_{0}<5$ & $t_{c t h}=168$ \\
\hline $5 \leq \mathrm{I}_{0}<10$ & $\mathrm{t}_{\mathrm{cth}}=\overline{96}$ \\
\hline $10 \leq \mathrm{I}_{0}<30$ & $t_{\mathrm{cth}}=48$ \\
\hline $30 \leq \mathrm{I}_{0}<60$ & $t_{c t h}=24$ \\
\hline $60 \leq \mathrm{l}_{0}<120$ & $t_{\mathrm{cth}}=8$ \\
\hline $120 \leq \mathrm{I}_{0}<240$ & $\mathrm{t}_{\mathrm{clh}}=4$ \\
\hline $\mathrm{I}_{0} \geq \overline{240}$ & $\mathrm{t}_{\mathrm{cth}}=2$ \\
\hline
\end{tabular}

Le coefficient de diffusion est déterminé par l'expression suivante [15]:

$$
\begin{aligned}
& D=\frac{R \cdot T \cdot L}{z \cdot F \cdot U} \cdot \frac{x_{d}-\alpha \cdot \sqrt{x_{d}}}{t} \\
& \alpha=2 \cdot \sqrt{\frac{R \cdot T \cdot L}{z \cdot F \cdot U}} \cdot e^{-i} \cdot\left(1-\frac{2 \cdot c_{d}}{c_{0}}\right)
\end{aligned}
$$

Où:

D: coefficient de diffusion, $\mathrm{m}^{2} / \mathrm{s}$;

$z$ : valeur absolue de valence du ion, pour les ions de chlore $\mathrm{z}=1$;

F: paramètre de Faraday, $F=9,648 \times 10^{4} \mathrm{~J} /(\mathrm{V} . \mathrm{mol})$;

$U$ : valeur absolue de la différence de potentiel, $\mathrm{V}$;

$\mathrm{T}$ : température de la solution, $\mathrm{K}$;

L: épaisseur de l'éprouvette, $\mathrm{m}$;

$x_{d}$ : profondeur de pénétration, $\mathrm{m}$;

t: durée de l'essai, s;

$\operatorname{erf}^{-l}$ : l'inverse de la fonction d'erreur;

$c_{d}:$ concentration des ions chlore avec laquelle la couleur est modifiée, $c_{d} \approx 0,07 \mathrm{~N}$;

$c_{\theta}$ : concentration des ions chlore dans la cellule, $\mathrm{N}$.

\subsection{L'essai de résistivité électrique}

La résistivité électrique est déterminée par la lecture initiale du courant dans l'essai de diffusion des ions chlore. 
La loi d'Ohm a été utilisée pour estimer les valeurs de la résistivité.

$R=\frac{V}{I}$

$R=\rho \cdot \frac{L}{A}$

$\rho=R \cdot \frac{A}{L}=\frac{V \cdot A}{L \cdot I}$

Où:

$\mathrm{R}$ : résistance électrique, $\Omega$;

I: intensité de courant, A;

$\mathrm{V}$ : différence de potentiel, $\mathrm{V}$;

$\rho$ : résistivité électrique, $\Omega . \mathrm{cm}$;

L: longueur, cm;

A: aire, $\mathrm{cm}^{2}$.

\subsection{L'essai d'absorption d'eau par capillarité}

L'essai d'absorption d'eau par capillarité a été réalisé sur des éprouvettes cylindriques de $105 \mathrm{~mm}$ de diamètre et $155 \mathrm{~mm}$ de hauteur, selon la norme E393 [16].

La pente de la droite qui s'ajuste le mieux avec les points expérimentaux, en fonction de la racine carrée du temps $\sqrt{t}$, représente le coefficient d'absorption d'eau par capillarité. Les résultats sont exprimés en $\mathrm{g} / \mathrm{m}^{2}$ pour chaque temps $\sqrt{t}$.

\section{L'ANALYSE DES RÉSULTATS}

Pour une évaluation plus efficace, les résultats on été analysés en deux séries de cinq mélanges chacune. La série A contient les mélanges nommées C300, C30M10, C330, C33M10 et C400. La série B contient les mélanges C330, C33M10, C33M15, C33SF10 et C33HRM10.

L'efficacité des additions actives a été mesurée à l'aide du facteur K, où

$K=\frac{C-X}{C} \times 100$

où,

$\mathrm{K}$ : Efficacité des additions actives, \%;

$\mathrm{C}$ : Valeur obtenue avec le mélange de référence;

$\mathrm{X}$ : Valeur obtenue avec le mélange $\mathrm{X}$.

\subsection{Résistance à lá compression}

La Fig. 2 représente l'évolution des résistances à la compression avec l'âge des mélanges de la série $\mathrm{A}$.

En analysant les résultats des résistances à la compression, il est possible de faire les constatations suivantes :

A cause de son contenu en ciment, le béton C400 est celui qui présente, pour tous les âges, les résistances les plus élevées.

Avec la même quantité de liant la substitution de $10 \%$ de ciment CEM I par du métakaolin a augmenté les résistances des bétons. Le béton avec $300 \mathrm{~kg} / \mathrm{m}^{3}$ de liant a eu une augmentation de $8 \%$ de sa résistance à 28 jours et de $21 \%$ à 90 jours. Le béton dont le contenu de liant était de $330 \mathrm{~kg} / \mathrm{m}^{3}$ a présenté une résistance à 7 jours inférieure à celui avec $300 \mathrm{~kg} / \mathrm{m}^{3}$, mais à l'âge de 28 jours l'efficacité est de $10 \%$ augmentant pour $18 \%$ à l'âge de 90 jours entre les bétons contenant du métakaolin.

Les résultats plus élevés présentés par le béton C400 ont été réduits par rapport à ceux du béton $\mathrm{C} 33 \mathrm{M} 10$ au fur et à mesure que l'âge augmentait, au point d'avoir une différence d'à peine $2 \mathrm{MPa}$ à l'âge de 90 jours. Il faut remarquer la forte différence de dosage en ciment entre ces deux mélanges, soit $100 \mathrm{~kg} / \mathrm{m}^{3}$ de plus pour le mélange $\mathrm{C} 400$.

Dans le but de comparer l'efficacité du métakaolin produit avec la microsilice SF et le HRM, la Fig. 3 montre l'évolution des résistances à la compression de la série $\mathrm{B}$.

Les valeurs obtenues, à l'âge de 7 jours, pour les mélanges $\mathrm{C} 33 \mathrm{M} 10$ et $\mathrm{C} 33 \mathrm{M} 15$ sont inférieures à celle du béton de référence, car il faut un temps d'induction pour que le métakaolin puisse réagir avec la chaux du ciment. Par contre, les valeurs obtenues sont plus élevées pour les âges les plus avancés. L'évolution des résistances de ces mélanges avec l'âge est similaire. Les résultats indiquent que la substitution de $15 \%$ du ciment par le métakaolin est plus avantageuse, car le mélange C33M15 a eu une

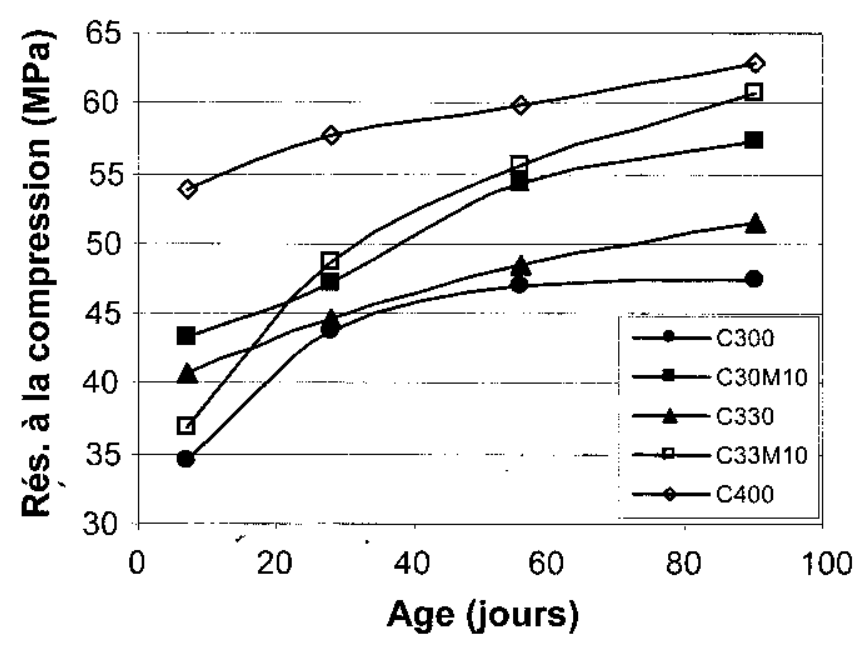

Fig. 2 - Série A : L’évolution des résistances à la compression.

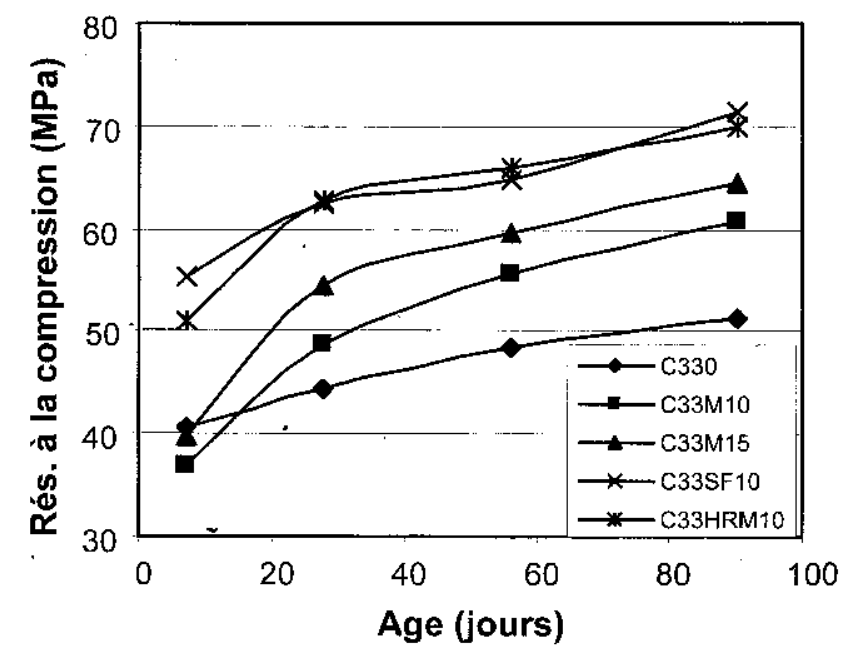

Fig. 3 - Série B : L'évolution des résistances à la compression. 
efficacité de $23 \%$ à l'âge de 28 jours et de $26 \%$ à l'âge de 90 jours.

Les SF et HRM sont les additions qui conduisent aux meilleurs résultats. En utilisant la fumée de silice, la valeur la plus élevée correspond à la résistance à l'âge de 7 jours, soit $55,3 \mathrm{MPa}$. À l'âge de 90 jours les résultats sont similaires à ceux obtenus avec le HRM, correspondant à une efficacité de l'ordre de $40 \%$.

\subsection{La diffusion des ions chlore}

Le Tableau 5 montre les moyennes des résultats obtenus dans les essais réalisés avec la série A. La Fig. 4 montre l'évolution avec l'âge du coefficient de diffusion du chlore.

Les résultats obtenus montrent que l'utilisation du métakaolin produit est largement bénéfique dans la réduction du coefficient de diffusion des ions chlore. Les valeurs pour les bétons incorporant le métakaolin sont, à tout âge, au moins $50 \%$ inférieures à celle du béton de référence.

La Fig. 4 témoigne du grand avantage de l'utilisation du métakaolin dans l'objectif de diminuer le coefficient de diffusion des ions chlore. Même pour le béton avec 400 $\mathrm{kg} / \mathrm{m} 3$ de ciment, les résultats sont inférieurs à ceux où le métakaolin était présent.

Les moyennes des résultats des essais réalisés avec les mélanges de la série B sont présentées au Tableau 6. La Fig. 5 montre également l'évolution avec l'âge des coefficients de diffusion des ions chlore.

Cet essai est suffisamment sensible pour mettre en évidence l'évolution du coefficient de diffusion des ions chlore avec l'augmentation en âge, principalement pour les

\begin{tabular}{|c|c|c|c|c|c|c|}
\hline \multicolumn{2}{|c|}{ Tableau 5 - Série A : Coefficients de diffusion du } \\
chlore $\left[\mathrm{m}^{2} / \mathbf{s}\right]$ \\
\hline $\begin{array}{c}\text { Mélange } \\
\text { / Âge }\end{array}$ & 28 & 56 & 90 & \multicolumn{3}{|c|}{$\begin{array}{l}\text { Efficacité des } \\
\text { additions actives K }\end{array}$} \\
\cline { 5 - 8 } & & & & 28 & 56 & 90 \\
\hline C300 & 37,57 & 35,99 & 27,63 & 0 & 0 & 0 \\
\hline C30M10 & 17,37 & 11,23 & 11,83 & -54 & -69 & -57 \\
\hline C330 & 33,44 & 24,77 & 21,07 & 0 & 0 & 0 \\
\hline C33M10 & 15,49 & 12,09 & 9,26 & -54 & -51 & -56 \\
\hline C400 & 38,13 & 30,84 & 22,25 & 0 & 0 & 0 \\
\hline
\end{tabular}

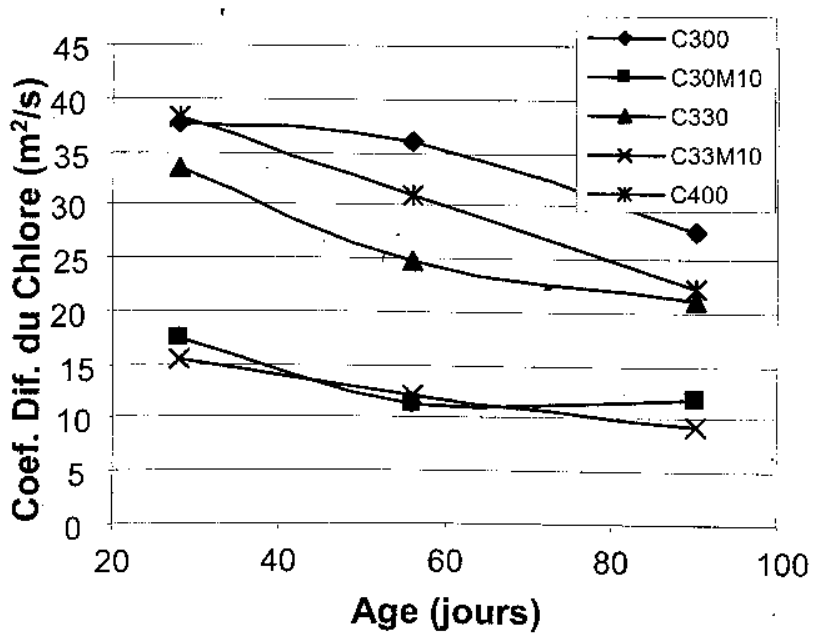

Fig. 4 - Série A : Évolution du coefficient de diffusion des ions chlore. bétons de qualité inférieure. Il exprime aussi la différence entre la qualité des bétons aux mêmes âges.

La substitution de $15 \%$ de métakaolin conduit à des résultats relativement meilleurs si ils sont comparés à ceux obtenus avec la substitution de $10 \%$. Donc il est clair que, le remplacement de $15 \%$ de ciment par le métakaolin est avantageux. A l'âge de 28 jours la composition C33M15 a atteint une diminution du coefficient de diffusion du chlore de $70 \%$ et de $65 \%$ à l'âge de 90 jours.

L'utilisation du SF et du HRM conduit à des résultats analogues à l'âge de 28 jours, la diminution du coefficient de diffusion du chlore est de $80 \%$. Pour le HRM cette valeur est maintenue avec l'augmentation en âge, néanmoins pour le SF la valeur se réduit à $70 \%$ à l'âge de 90 jours.

\subsection{Résistivité électrique}

Les résultats des essais de résistivité électrique obtenus pour la série A sont présentés au Tableau 7. La Fig. 6 montre l'évolution de la résistivité avec l'âge du béton.

Les valeurs obtenues montrent que l'utilisation du métakaolin améliore de manière effective le comportement du béton. La différence, qui se maintient à tout âge, pour les valeurs données par les bétons avec métakaolin, supérieures à $80 \%$, est très importante. De ce fait, l'essai montre clairement la différence de qualité entre les bétons à n'importe quel âge. A plus long terme, la différence d'ordre de $17 \%$ est perceptible avec 1'augmentation du contenu de ciment dans les bétons.

Le Tableau 8 montre les résultats de l'essai de l'évolution de la résistivité pour la série A. La Fig. 7 groupe

\begin{tabular}{|c|c|c|c|c|c|c|}
\hline \multicolumn{6}{|c|}{ Tableau 6 - Série B : Coefficients de diffusion du } \\
chlore $\left.\mathbf{~ m}^{2} / \mathbf{s}\right]$ \\
\hline $\begin{array}{c}\text { Mélange / } \\
\text { Âge }\end{array}$ & 28 & 56 & 90 & \multicolumn{3}{|c|}{$\begin{array}{l}\text { Efficacité des } \\
\text { additions actives K }\end{array}$} \\
\cline { 4 - 8 } & & & & 28 & 56 & 90 \\
\hline C330 & 33,44 & 24,77 & 21,07 & 0 & 0 & 0 \\
\hline C33M10 & 15,49 & 12,09 & 9,26 & -54 & -51 & -56 \\
\hline C33M15 & 10,17 & 6,84 & 7,27 & -70 & -72 & -65 \\
\hline C33SF10 & 6,36 & 5,73 & 6,03 & -81 & -77 & -71 \\
\hline C33HRM10 & 5,63 & 4,57 & 3,83 & -83 & -82 & -82 \\
\hline
\end{tabular}

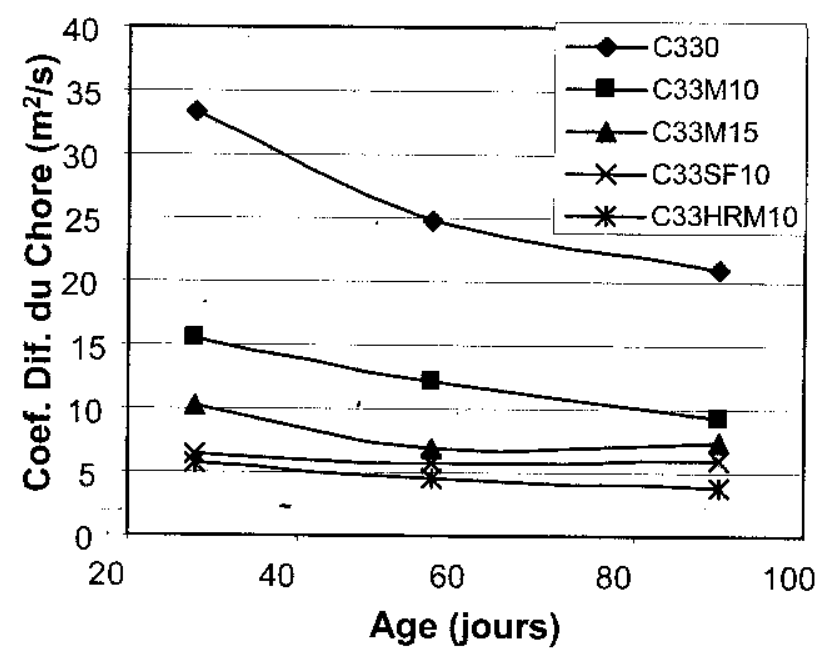

Fig. 5 - Série B : Évolution des coefficients de diffusion du chlore. 


\begin{tabular}{|c|c|c|c|c|c|c|}
\hline \multicolumn{7}{|c|}{ vité électrique $[\Omega . \mathrm{cm}]$} \\
\hline \multirow{2}{*}{$\begin{array}{l}\text { Mélange } \\
\text { / Âge }\end{array}$} & \multirow{2}{*}{28} & \multirow[t]{2}{*}{56} & \multirow{2}{*}{90} & \multicolumn{3}{|c|}{$\begin{array}{l}\text { Efficacité des } \\
\text { additions actives K }\end{array}$} \\
\hline & & & & 28 & 56 & 90 \\
\hline $\mathrm{C} 300$ & 0,43 & 0,41 & 0,45 & 0 & 0 & 0 \\
\hline $\mathrm{C} 30 \mathrm{M} 10$ & 0,79 & 0,99 & 1,09 & 84 & 140 & 144 \\
\hline $\mathrm{C} 330$ & 0,38 & 0,51 & 0,58 & 0 & 0 & 0 \\
\hline C33M10 & 0,66 & 0,82 & 1,28 & 71 & 60 & 119 \\
\hline $\mathrm{C} 400$ & 0,35 & 0,40 & 0,49 & 0 & 0 & 0 \\
\hline
\end{tabular}

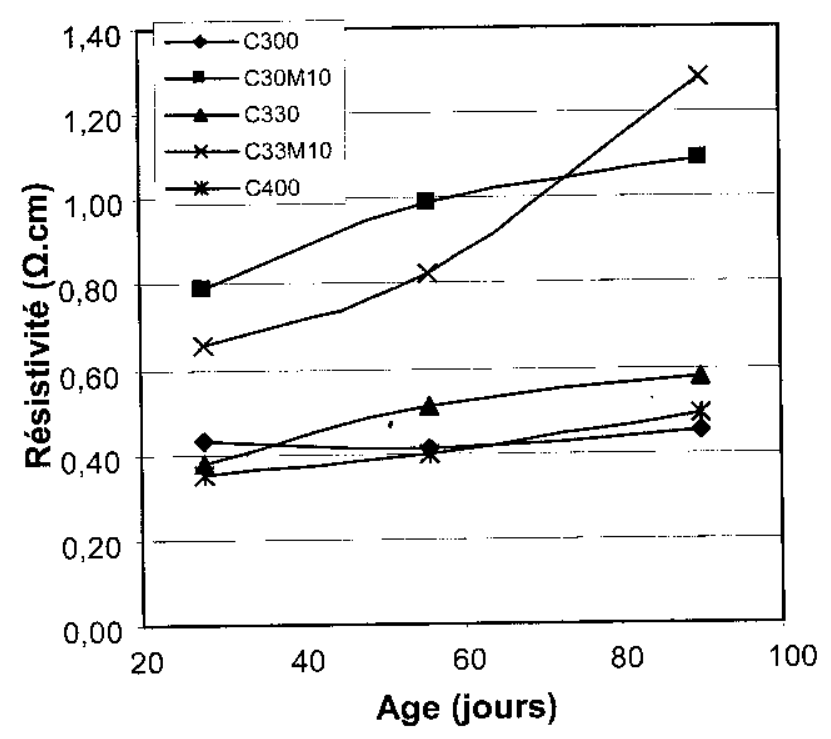

Fig. 6 - Série A : Évolution de la résistivité électrique.

l'évolution de la résistivité électrique avec l'âge des bétons.

Les résultats, les plus intéressants, ont été observés avec la substitution de $15 \%$ de métakaolin. À l'âge de 28 jours l'augmentation de la résistivité est de $142 \%$, valeur qui augmente à $162 \%$ à l'âge de 90 jours. Les résultats obtenus avec SF et HRM sont à peu près identiques, avec une différence plus importante pour le HRM à l'âge de 90 jours. Avec le HRM, l'efficacité est $220 \%$ à 28 jours relativement au béton de référence, cette valeur augmente à $230 \%$ à 90 jours.

\subsection{Absorption d'eau par capillarité}

Le Tableau 9 présente les valeurs obtenues pour les bétons de la série A et la Fig. 8 montre l'évolution avec l'âge du béton du coefficient de capillarité.

À tous les âges, les bétons incorporant du métakaolin ont les coefficients d'absorption d'eau les plus faibles. Le béton C300, comparativement au béton C 330 , se comporte le mieux. Cela est dû au rapport eau/liant plus bas, qui a été adopté pour ce mélange.

Cet essai indique aussi que l'utilisation du métakaolin est très positive dans l'amélioration de la performance du béton. Néanmoins, on constate que l'augmentation de l'âge du béton diminue la différence entre les bétons avec métakaolin et celui de référence.

A l'âge de 90 jours le béton C30M10 présente un coefficient d'absorption d'eau $8 \%$ plus faible que celui du béton C300. La substitution de $10 \%$ de métakaolin dans le

\begin{tabular}{|c|c|c|c|c|c|c|}
\hline \multicolumn{7}{|c|}{ 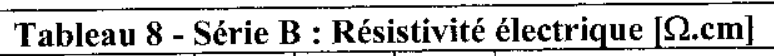 } \\
\hline \multirow{2}{*}{$\begin{array}{c}\text { Mélange / } \\
\text { Âge }\end{array}$} & \multirow{2}{*}{28} & \multirow{2}{*}{56} & \multirow[t]{2}{*}{90} & \multicolumn{3}{|c|}{$\begin{array}{l}\text { Efficacité des } \\
\text { additions actives } \mathrm{K}\end{array}$} \\
\hline & & & & 28 & 56 & 90 \\
\hline $\mathrm{C} 33$ & 0,38 & 0,51 & 0,58 & 0 & 0 & 0 \\
\hline C33M10 & 0,66 & 0,82 & 1,28 & 71 & 60 & 119 \\
\hline $\mathrm{C} 33 \mathrm{M} 15$ & 0,93 & 1,23 & 1,53 & 142 & 139 & 162 \\
\hline C33SF 10 & 1,15 & 1,5 & 1,40 & 199 & 208 & 140 \\
\hline C33HRM10 & 1,23 & 1,69 & 1,93 & 219 & 229 & 230 \\
\hline
\end{tabular}

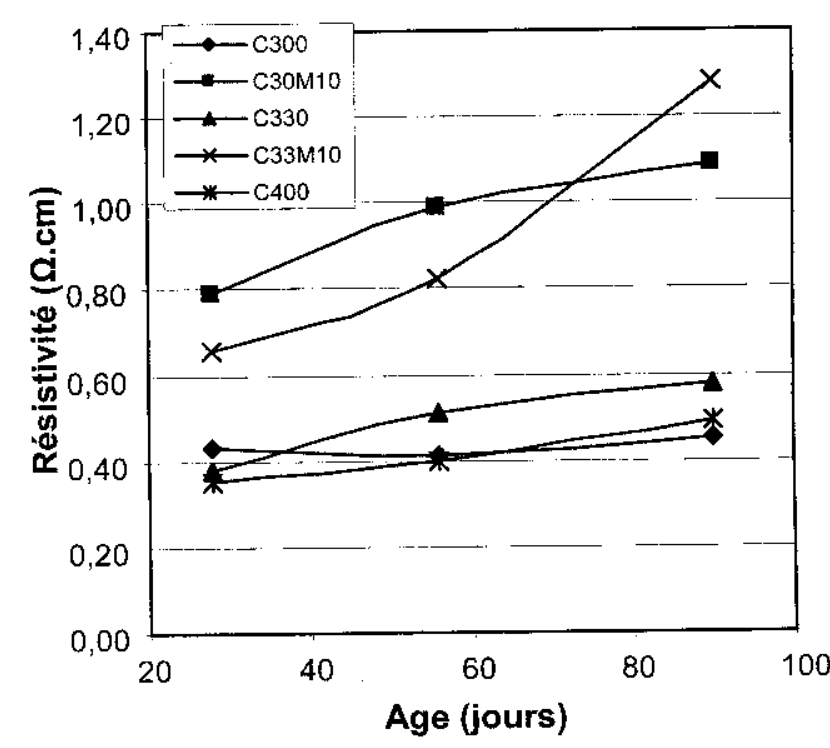

Fig. 7 - Série B : Évolution de la résistivité électrique.

mélange C33M10 donne une efficacité de 35\% par rapport au béton de référence.

Le Tableau 10 montre les valeurs obtenues pour les bétons de la série B et la Fig. 9 montre l'évolution avec l'âge du béton du coefficient de capillarité.

Avec 10 et $15 \%$ de substitution de ciment par le métakaolin, l'amélioration est assez significative, soit $60 \%$ à 28 jours, valeur qui diminue à $35 \%$ à 90 jours. Il n'y a pas de différence significative entre l'ajout de $10 \mathrm{ou} 15 \%$. Avec l'utilisation du SF et HRM les améliorations sont encore plus importantes. A l'âge de 28 jours l'amélioration est de $75 \%$, qui diminue pour atteindre $60 \%$ à 90 jours.

L'effet du rapport eau/liant est analysé en comparant les résultats des essais des mélanges $\mathrm{C} 300(\mathrm{E} / \mathrm{C}=0,35)$ et $\mathrm{C} 330$ $(\mathrm{E} / \mathrm{C}=0,45)$, ainsi que entre les mélanges $\mathrm{C} 30 \mathrm{M} 10$ $(\mathrm{E} / \mathrm{C}=0,35)$ et $\mathrm{C} 33 \mathrm{M} 10(\mathrm{E} / \mathrm{C}=0,45)$. Une augmentation de l'absorption d'eau par capillarité supérieure à $30 \%$ a été constatée dans le groupe $\mathrm{C} 300$ et $\mathrm{C} 330$ due au rapport $\mathrm{E} / \mathrm{C}$ plus élevé. Entre les mélanges C30M10 e C33M10 cette augmentation a été réduite et à long terme cette réduction est de l'ordre de $5 \%$.

En ce qui concerne les résistances à la compression, aucune différence significative n'a été vérifiée à l'intérieur des deux groupes de mélanges. Finalement, on peut conclure que le rapport $\mathrm{E} / \mathrm{C}$ plus faible n'a pas influencé favorablement les résultats des essais de diffusion du chlore et de la résistivité électrique. Ces conclusions sur l'effet du rapport $\mathrm{E} / \mathrm{C}$ sont considérées valables, car si l'augmentation 


\begin{tabular}{|c|c|c|c|c|c|c|}
\hline \multicolumn{6}{|c|}{ Tableau 9- Série A : Coefficients d'absorption d'eau } \\
par capillarité \\
\hline $\begin{array}{c}\text { Mélange } \\
\text { / Âge }\end{array}$ & 28 & 56 & 90 & \multicolumn{3}{|c|}{$\begin{array}{l}\text { Efficacité des } \\
\text { additions actives K }\end{array}$} \\
\cline { 4 - 8 } & & & & 28 & 56 & 90 \\
\hline C300 & 241,7 & 173,3 & 127,0 & 0 & 0 & 0 \\
\hline C30M10 & 122,5 & 143,3 & 116,7 & -49 & -17 & -8 \\
\hline C330 & 356,8 & 222,9 & 171,3 & 0 & 0 & 0 \\
\hline C33M10 & 146,4 & 122,5 & 111,2 & -59 & -45 & -35 \\
\hline C400 & 206,9 & 166,1 & 144,1 & 0 & 0 & 0 \\
\hline
\end{tabular}

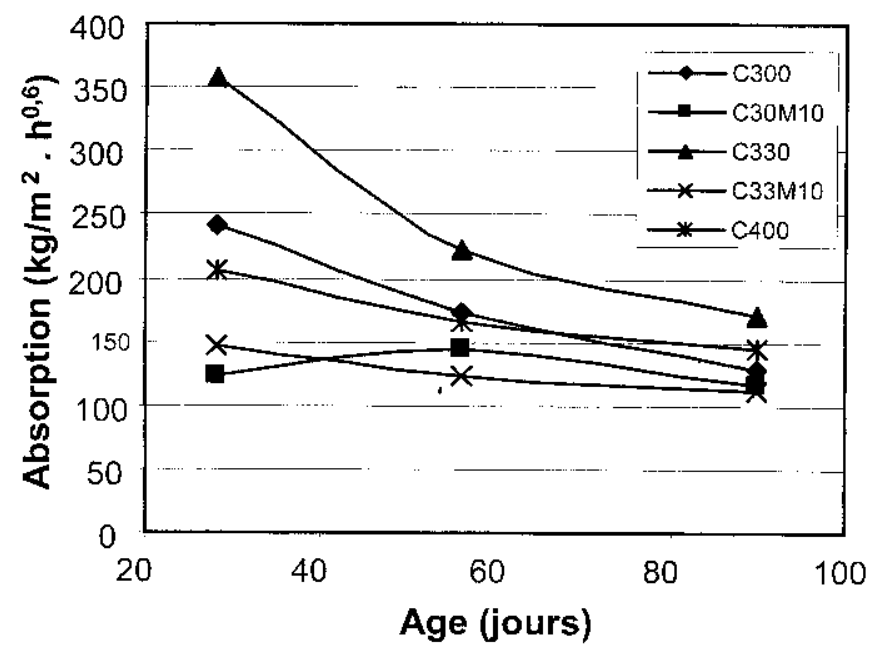

Fig. 8 - Séric A : Évolution des coefficients d'absorption d'eau.

du contenu de liant entre les mélanges comparés a été de l'ordre de $10 \%$; la réduction du rapport E/C a été de $20 \%$.

\section{CONCLUSIONS}

Les résultats obtenus dans ce travail nous amènent aux conclusions suivantes:

1. Pour la transformation du kaolin en métakaolin, une température de $750^{\circ} \mathrm{C}$ est nécessaire. Ce procédé implique une perte de $12 \%$ en masse. Tous les matériaux doivent atteindre cette température.

2. C'est principalement avec les essais qui évaluent la durabilité du bétón que les résultats démontrent les avantages de la substitution partielle de ciment par le métakaolin.

3. Il est possible de réduire la quantité de ciment pour obtenir des bétons équivalents lors de l'addition du métakaolin. Cette rẻduction dépend de l'importance de la résistance désirée.

4. Le remplacement de $15 \%$ de ciment par du métakaolin est le plus efficace, autant pour la résístance que pour la durabilité.

5. Les meilleurs résultats obtenus avec le métakaolin de haute réactivité (HRM) sont probablement associés à la pureté de la matière première et à la plus faible dimension de ses particules.

6. Les résultats obtenus avec SF sont semblables à ceux des HRM.

7. D'après les résultats obtenus dans cette étude on peut conclure qu'il est possible, au Portugal, de produire des

\begin{tabular}{|c|c|c|c|c|c|c|}
\hline \multicolumn{6}{|c|}{ Tableau 10 - Série B : Coefficients d'absorption d'eau } \\
par capillarité \\
\hline $\begin{array}{c}\text { Mélange / } \\
\text { Âge }\end{array}$ & 28 & 56 & 90 & \multicolumn{3}{|c|}{$\begin{array}{c}\text { Efficacité des } \\
\text { additions actives K }\end{array}$} \\
\cline { 4 - 7 } & & & & 28 & 56 & 90 \\
\hline C330 & 356,8 & 222,9 & 171,3 & 0 & 0 & 0 \\
\hline C33M10 & 146,4 & 122,5 & 111,2 & -59 & -45 & -35 \\
\hline C33M15 & 129,5 & 85,0 & 109,1 & -64 & -62 & -36 \\
\hline C33SF10 & 93,4 & 76,0 & 81,0 & -74 & -66 & -53 \\
\hline C33HRM10 & 84,8 & 77,6 & 72,5 & -76 & -65 & -58 \\
\hline
\end{tabular}

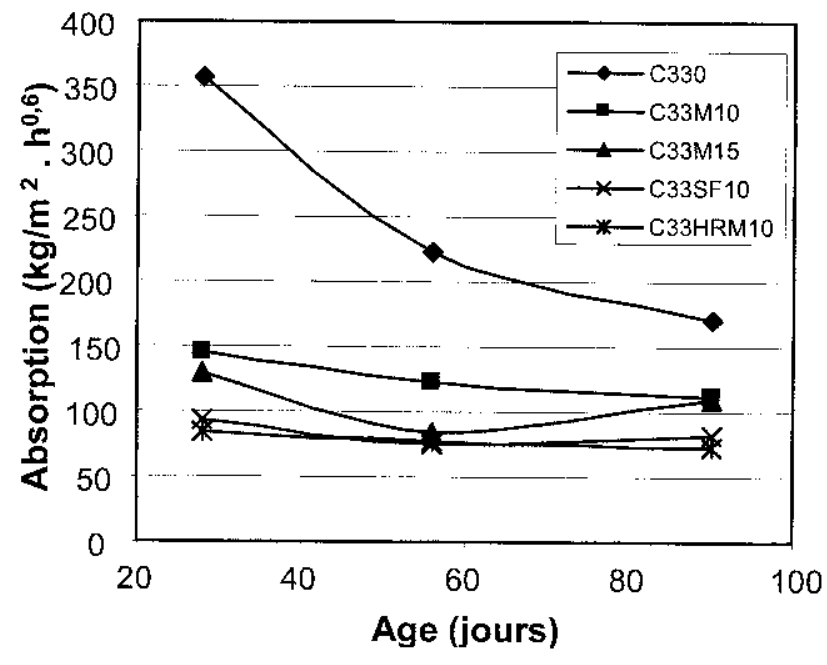

Fig. 9 - Série B : Évolution des coefficients d'absorption d'eau.

bétons écologiquement efficaces. Néanmoins, pour la production de métakaolin à l'échelle industrielle, une étude de l'optimalisation de calcination du kaolin, en fonction du type de four employé, s'avère nécessaire.

\section{REMERCIEMENTS}

Ce travail a été réalisé dans le cadre du Project POCTI/ECM/34338/99, financé par la « Fundação para a Ciência e a Tecnologia » que les auteurs remercient.

\section{REFERENCES}

[1] Caldarone; M.A, Gruber, K.A and Burg, R.G., 'Highreactivity metakaolin: a new generation mineral admixture', Concrete International 16 (11) (1994) 37-40.

[2] Marsh D., 'An alternative to silica fume', Concrete Production 97 (11) (1994) 24-30.

[3] Wild, S.; Khatib, J. and Roose, LJ., 'Chemical and autogenous shrinkage or Portland cement-metakaolin pastes', Advanced Cement Research 10 (3) (1998) 109-119.

[4] Rols, S., Mbessa, M., 'Ambroise, J. and Péra, J., 'Influence of ultra-fine particle type on properties of very-high-strength concrete', in Malhotra, V.M., Helene, P., Prudencio, L.R., Dal Mọlin, D.C.C., editors, Proceedings of the Second CANMET/ACI International Conference on High Performance Concrete and Performance and Quality of Concrete Structures, Gramado, RS, Brazil, 1999, 671-686. 
[5] Marwan, T., Péra, J. and Ambroise, J., 'The action of some aggressive solutions on Portland and calcined laterite blended cement concretes', in: Malhotra V.M, editor. Proceedings of the Fourth International Conference on Fly Ash, Silica fume, Slag and Natural Pozzolans in Concrete. Vol I. Instanbul, Turkey, May 1992, 763-779.

[6] Bosc, J.L., Kouame, K. and Péra, J., 'Improvement of concrete durability in tropical marine environment by adding métakaolin and superplasticisers', in Proceedings of the Sixth International Conference on Durability of Building Materials and Components, Vol. 1. Omiya, Japan, 1993, 448-457.

[7] Khatib, J. and Wild, S., 'Sulphate resistance of metakaolin mortar', Cement Concrete Research 28 (1) (1998) 83-92.

[8] Péra, J., Rols, S., Chabannet, M. and Ambroise, J., 'Influence of the cement type on the resistance of concrete to an agricultural environment', in: Cohen, M., Mindess, S., Skalny, J., editors. Materials Sciences of Concrete: The Sidney Diamond Symposium (The American Concrete Society, 1998) 419-430.

[9] Sabir, B.B., Wild, S. and Bai, J., 'Metakaolin and calcined clays as pozzolans for concrete: a review', Cement \& Concrete Composites 23 (2001) 441-454.
[10] Dunster, A.M, Parsonage, J.R. and Thomas, M.J.K., 'The pozzolanic reaction of metakaolin and its effects on PC hydration', Journal of Materials Science 28 (1993) 13451350.

[11] Coutinho, A.S. and Gonçalves, A., 'Production and properties of Concrete' (LNEC, Lisbon, 1994) [only available in Portuguese].

[12] Liu, J.N., 'Strength and hydration of an activated aluminosilicate material', Pennsylvania State University, USA, 1998.

[13] Faury, J., 'Le béton. Influence de ses constituants inertes. Règles à adopter pour sa meilleure composition. Sa confection et son transport sur les chantiers' (Dunod, Paris, 1958).

[14] LNEC - E226, 'Concrete: Compression Test', LNEC, Lisboa, 1968 [only available in Portuguese].

[15] Luping, T., 'Chloride transport in concrete - Measurement and prediction', Doctoral Thesis, Chalmers University of Technology, Gothenburg, Sweden, 1996.

[16] LNEC - E393, 'Concretes: Determination of capillarity water absorption', LNEC, Lisbon, 1993 [only available in Portuguese]. 


\section{MATERIALS AND STRUCTURES MATERIAUX ET CONSTRUCTIONS}

\section{CONTENTS}

\section{Volume 38, Issue 277, April 2005}

\section{Scientific reports}

289 Fatigue behavior of concrete subjected to biaxial loading in the compression region B. Mu and S. P. Shah

299 Mechanical threshold of cementitious materials at early age J. M. Torrenti and F. Benboudjema

305 Mode I and mixed mode fracture studies in brittle materials using the Brazilian disc specimen H. N. Atahan, M. A. Tasdemir, C. Tasdemir, N. Ozyurt and S. Akyuz

A comparison of transport properties for concrete using the ponding test and the accelerated chloride migration test C. C. Yang

Durability of bonded cement-based overlays: effect of metal fibre reinforcement A. Turatsinze, J.-L. Granju, V. Sabathier and H. Farhat

Flexural behavior of reinforced concrete beams with cementitious repair materials S. K. Park and D.-S. Yang

Mechanical behaviour of thermally damaged high-strength steel fibre reinforced concrete G. M. Giaccio and R. L. Zerbino

343 Shear behaviour of steel fibre reinforced concrete beams A. Meda, F. Minelli, G. A. Plizzari and P. Riva

Effect of normalizing heat treatment on the mechanical behaviour of low-alloy steel weld metals V. B. Trindade, J. C. Payäo-Filho, A. S. Guimarães and R. P. R. Paranhos

Preliminaty modeling of internal heterogeneities in fibre reinforced concrete

O. Plé and O. Bayard

Influence of the pozzolanic fraction obtained from vitrified bottom-ashes from MSWI on the properties of cementitious composites

A. Saccani, F. Sandrolini, F. Andreola, L. Barbieri, A. Corradi and I. Lancelloti

M. Berra, T. Mangialardi and A.E. Paolini

$381 \quad$ Alkali-aggregate reaction in Swiss tunnels

A. Leemann, C. Thalmann and W. Studer

Influence of composite materials confinement on alkali aggregate expansion 1. Mohamed, L. Curtil, S. Ronel-Idrissi and P. Hamelin

J.S. Chen, S. Y. Wong and K. -Y. Lin

L'emploi de métakaolin dans la production de béton écologiquement efficace (The use of metakaolin to produce the eco-efficient conctete)

L. A. P. Oliveira, S. Jalali, J. M. Fernandes et E. Torres

Study on hydration of Portland cement with fly ash using electrical measurement

$X$. Wei and Z. $\mathrm{Li}$

Dama de Elche: Pigments, surface coating and stone of the sculpture

M. P. Luxán, J. L. Prada and F. Dorrego 